\title{
Abell Clusters as Tracers of Large-Scale Structure: Alignments and Substructure
}

\author{
G. Rhee \& P. Katgert \\ Sterrewacht Leiden
}

Binggeli (A \& A, 107,338,1982) showed that neighbouring Abell clusters with pair distances less than 30 to $\mathbf{4 0} h_{50}^{-1} \mathrm{Mpc}$ show directional correlation. Binggeli's result is based on structure data of 44 Abell clusters (all with $z_{\text {spectr }}<0.1$ ). Binggelli's result was questioned by Struble and Peebles (A.J., 90, 582,1985). They produce a visual estimate of the direction of cluster elongation for 237 clusters. They did not observe reduced probability for small D large $\Theta$ pairs.

We have explained the disagreement in position angles of individual clusters for the 37 clusters that Binggeli and Struble and Peebles sample have in common. We find that the discrepancy between position angles of individual clusters can be explained in terms of cluster elongation strengths. The position angle differences are smaller for the more elongated clusters (Rhee and Katgert, A \& A in press).

We have repeated Binggeli's test. Our sample consists of all 108 Abell clusters, richness class $>1,9^{h} \leq \alpha \leq 18^{h} .5, \mathrm{z}_{\mathrm{spectr}} \leq 0.1$. From our sample of Abell clusters we find the effect to be weaker than that found by Binggeli. The Binggeli effect seems more pronounced for those members of our sample that are members of Binggeli's sample.

We are currently studying substructure in 2-D galaxy distributions in Abell clusters. We are using the Lee statistic as well as percolation as measures of substructure. We do not find as much substructure as Geller and Beers (P.A.S.P., 94, 421).

We also studying working on aligments of the elongation of galaxies in clusters and overall elongation of the clusters. For a sample of 37 clusters studied by Binggeli and Struble \& Peebles we find a good alignment of first-ranked galaxy p.a. with cluster p.a. We are working on alignments of the 20 brightest galaxies with each other and with their cluster for a sample of 108 Abell clusters. We select the 20 brightest galaxies whithin $2 \mathrm{Mpc}$ of the cluster center. We find three categories of cluster:

i) alignment of the brightest galaxies among themselves, and with the cluster p.a.

ii) alignment of the brightest cluster galaxies among themselves but not with the cluster p.a. as determined from the 100 brightest galaxies.

iii) no alignments are present. 